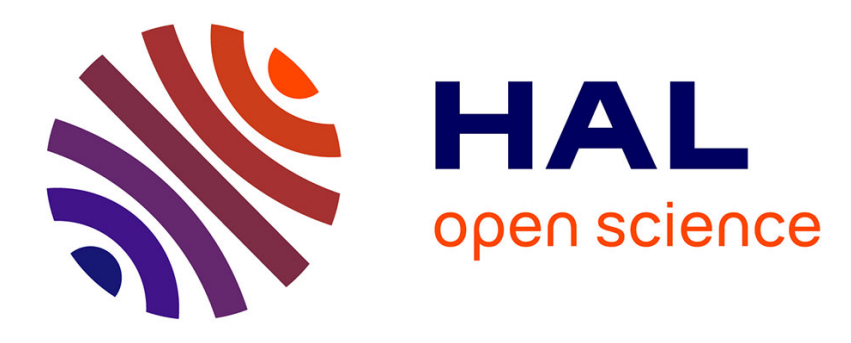

\title{
On the classical two-dimensional one-component Coulomb plasma
}

\author{
A. Alastuey, B. Jancovici
}

\section{To cite this version:}

A. Alastuey, B. Jancovici. On the classical two-dimensional one-component Coulomb plasma. Journal de Physique, 1981, 42 (1), pp.1-12. 10.1051/jphys:019810042010100 . jpa-00208987

\section{HAL Id: jpa-00208987 https://hal.science/jpa-00208987}

Submitted on 1 Jan 1981

HAL is a multi-disciplinary open access archive for the deposit and dissemination of scientific research documents, whether they are published or not. The documents may come from teaching and research institutions in France or abroad, or from public or private research centers.
L'archive ouverte pluridisciplinaire HAL, est destinée au dépôt et à la diffusion de documents scientifiques de niveau recherche, publiés ou non, émanant des établissements d'enseignement et de recherche français ou étrangers, des laboratoires publics ou privés. 


\title{
LE JOURNAL DE PHYSIQUE
}

J. Physique 42 (1981) 1-12

JANVIER 1981, PAGE 1

Classification

Physics Abstracts

$05.20-63.70-50.00$

\section{On the classical two-dimensional one-component Coulomb plasma}

\author{
A. Alastuey $(*)$ and B. Jancovici $(*)$ \\ Institut Laue-Langevin, 156 X, 38042 Grenoble Cedex, France \\ (Reçu le 9 juillet 1980, accepté le 12 septembre 1980)
}

Résumé. - On étudie quelques aspects de la mécanique statistique d'équilibrè d'un plasma coulombien classique
à une composante en deux dimensions (l'interaction est logarithmique). On calcule exactement l'énergie libre pour
une valeur particulière de la température. A basse température, les particules forment un réseau triangulaire,
qui est stable; ses modes de vibration sont des plașmons longitudinaux et des phonons transverses de comporte-
ment normal. On étudie l'approximation harmonique autocohérente; elle n'a de solution pour la vitesse du son
transverse qu'à basse température. On compare les énergies libres obtenues par différentes méthodes. Il est très
vraisemblable que le modèle a une transition de phase solide-fluide.

Abstract. - We study some aspects of the equilibrium statistical mechanics of a classical two-dimensional onecomponent Coulomb plasma (the interaction is logarithmic). The free energy is calculated exactly, for one special value of the temperature. At low temperature, the particles form a triangular lattice, which is stable; its vibration modes are well-behaved longitudinal plasmons and transverse phonons. The self-consistent harmonic approximation is studied; it has a solution for the transverse sound velocity at low temperature only, a strong indication that the solid phase is unstable at high temperature. The free energies obtained in different ways are compared. It is very likely that the model has a solid-fluid phase transition.

1. Introduction. - A one-component plasma is a system of $N$ identical particles of charge $e$ and mass $m$ embedded in a uniform neutralizing background of opposite charge. In two dimensions, the Coulomb interaction potential between two particles at a distance $r$ from one another is

$$
v(r)=-e^{2} \ln (r / L),
$$

where $L$ is a scaling length ; the logarithmic form of this potential results from the requirement that it obeys the two-dimensional Poisson equation. Studying models with a logarithmic interaction is of interest for the understanding of real systems such as solids, magnets, superfluids, in which a logarithmic interaction between dislocations or vortices does occur. But the two-dimensional one-component Coulomb plasma is also a very interesting mathematical model by itself, mainly because some of its properties can be calculated exactly. The present paper deals with some aspects of the equilibrium classical statistical mechanics of this plasma.

(*) Permanent address : Laboratoire de Physique Théorique et Hautes Energies, Université de Paris-Sud, 91405 Orsay Cedex, France.
The equation of state is exactly known. It has been obtained by a scaling argument [1], resulting into

$$
p=\left(k_{\mathrm{B}} T-\frac{1}{4} e^{2}\right) \rho,
$$

where $p$ is the pressure, $k_{\mathrm{B}}$ Boltzmann's constant, $T$ the temperature, $\rho$ the number density. The pressure (1.2) becomes negative at low temperatures. This is not a special feature of the two-dimensional case; it is well known that the same phenomenon occurs for the three-dimensional one-component plasma. The negativeness of the pressure causes no special difficulty if the background is considered as rigidly maintained at a uniform constant density by some external constraint.

The knowledge of the pressure (1.2) does not however give the whole thermodynamical story, since the free energy is not fully known. One knows that the free energy per particle exists in the thermodynamic limit [2], and, from the scaling argument, that the excess free energy per particle must be of the form

$$
\frac{F_{\mathrm{exc}}}{N}=-\frac{e^{2}}{4} \ln \pi \rho L^{2}+f(T)
$$


but not exact expression is known for the function of the temperature $f(T)$. By integrating the pressure with respect to the density, one obtains nothing more than (1.3); the system does not behave like an ideal gas in the low-density limit, and therefore one cannot obtain more information by considering this limit. The separation of the density and temperature dependences in (1.3) is related to the fact that the dimensionless coupling constant for the potential (1.1), $\Gamma=e^{2} / k_{\mathrm{B}} T$, does not depend on the density.

The model has been shown to be crystalline [3], with a triangular lattice, at zero temperature. At finite temperatures, the model has been studied through approximation methods which are usually considered as appropriate for fluids : the Debye-Hückel approximation [4] on one hand, the resolution of an approximate integral equation for the pair correlation function $[5,6]$ on the other hand.

Although no phase transition is found within the above-mentioned approximations, it is an intriguing question to know whether the exact model has a phase transition (or perhaps several ones). Such a phase transition would be of a rather unusual type, since it might show up in the free energy (1.3) as a singularity in $f(T)$, while the equation of state would remain the smooth function (1.2).

The contributions of the present paper are as follows. On one hand, we give an exact calculation of the free energy for one special value of the temperature, namely at $k_{\mathrm{B}} T=e^{2} / 2$ (Sec. 2). On the other hand, we investigate the low-temperature behaviour. We first study the simple harmonic-solid model (Sec. 3) which is found to be stable, i.e. all phonon frequencies are real (of course this two-dimensional « solid» has the well-known feature of showing no long-range positional order). We then turn to the self-consistent harmonic approximation (Sec. 4), and find that there is no solution for the transverse sound velocity when the temperature is high enough, a strong indication that the solid becomes unstable. In the conclusion (Sec. 5), we compare the free energies obtained in different ways and give arguments in favour of the existence of a solid-fluid phase transition. The Appendix is a warning against an incorrect application of a standard theorem of statistical mechanics.

2. Exact free energy at $k_{\mathrm{B}} T=e^{2} / 2$. The free energy can be exactly calculated at this temperature through a method sketched by Deutsch et al. [4]. We consider $N$ particles in a circular background of radius $R$. Let $\mathbf{r}_{i}$ be the position of particle $i$, and $\mathbf{r}_{i j}=\mathbf{r}_{j}-\mathbf{r}_{i}$. The total potential energy is [3]

$$
\begin{aligned}
V=-\frac{3 N^{2} e^{2}}{8}+\frac{N e^{2}}{2} \ln \frac{R}{L} & +\frac{N e^{2}}{2} \sum_{i}\left(\frac{r_{i}}{R}\right)^{2}- \\
& -\frac{e^{2}}{2} \sum_{i>j} \ln \left(\frac{r_{i j}}{R}\right)^{2} .
\end{aligned}
$$

In terms of scaled variables $\mathbf{z}_{i}=\mathbf{r}_{i} / R$, the excess canonical partition function is, for $k_{\mathrm{B}} T=e^{2} / 2$,

$$
\begin{aligned}
Q_{\mathrm{exc}}=\exp & \left(\frac{3}{4} N^{2}-N \ln \frac{R}{L}\right) \int_{0}^{1} \frac{\mathrm{d}^{2} z_{1}}{\pi} \cdots \int_{0}^{1} \frac{\mathrm{d}^{2} z_{N}}{\pi} \times \\
& \times \exp \left(-N \sum_{i} z_{i}^{2}\right) \prod_{i>j}\left(\mathbf{z}_{j}-\mathbf{z}_{i}\right)^{2} .
\end{aligned}
$$

Let $\theta_{i}$ be the polar angle of $\mathbf{z}_{i}$ :

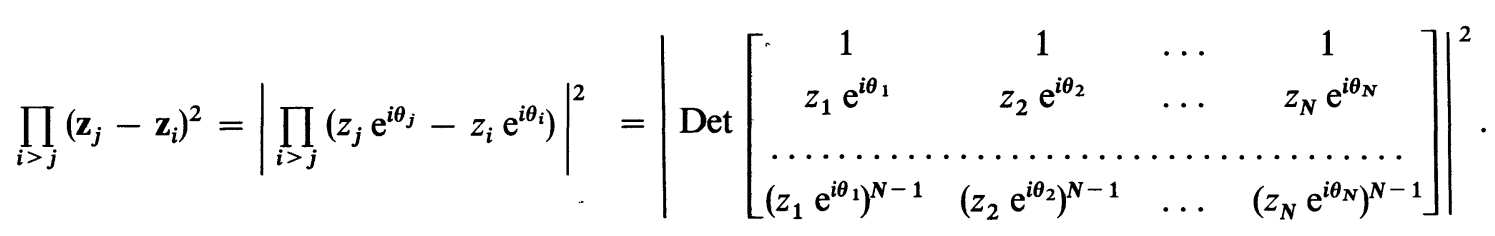

When the Vandermonde determinant in (2.3) is expanded, it yields $N$ ! terms which are mutually orthogonal with respect to integration upon all angles $\theta_{i}$. Therefore,

$$
\int_{0}^{2 \pi} \mathrm{d} \theta_{1} \cdots \int_{0}^{2 \pi} \mathrm{d} \theta_{N} \prod_{i>j}\left(\mathbf{z}_{j}-\mathbf{z}_{i}\right)^{2}=(2 \pi)^{N}\left[z_{1}^{0} z_{2}^{2} z_{3}^{4} \ldots z_{N}^{2(N-1)}+\cdots\right] ;
$$

there are $N$ ! terms in (2.4), which result from the explicitly written one by permutations of the indices. Each one of these terms gives the same contribution to (2.2). Using new integration variables $x_{i}=N z_{i}^{2}$, one finds

$$
Q_{\text {exc }}=\exp \left(\frac{3}{4} N^{2}-N \ln \frac{R}{L}\right) \frac{N !}{N^{N(N+1) / 2}} \int_{0}^{N} \mathrm{~d} x_{1} \mathrm{e}^{-x_{1}} \int_{0}^{N} \mathrm{~d} x_{2} x_{2} \mathrm{e}^{-x_{2}} \ldots \int_{0}^{N} \mathrm{~d} x_{N} x_{N}^{N-1} \mathrm{e}^{-x_{N}} .
$$

We now show that the upper bound $N$ of the integrals in (2.5) can be replaced by $+\infty$ in the thermodynamic limit. We write

$$
Q_{\mathrm{exc}}=\exp \left(\frac{3}{4} N^{2}-N \ln \frac{R}{L}\right) \frac{1 ! 2 ! \ldots N !}{N^{N(N+1) / 2}} Q^{\prime}
$$


and prove that

$$
\lim _{N \rightarrow \infty} \frac{1}{N} \ln Q^{\prime} \equiv \lim _{N \rightarrow \infty} \frac{1}{N} \sum_{n=0}^{N-1} \ln \left(1-\frac{1}{n !} \int_{N}^{\infty} \mathrm{e}^{-x} x^{n} \mathrm{~d} x\right)
$$

vanishes, as follows. First, we can discard in the sum in (2.7) the term $n=0$, since this single term gives no contribution to the limit. We group the remaining terms in two classes, $1 \leqslant n \leqslant N y$ and $N y<n \leqslant N-1$, where $y$ is some fixed number such that $0<y<1$. For computing the contribution of the first class, we use the inequalities

and

$$
n !>\mathrm{e}^{-n} n^{n} \quad(n \geqslant 1)
$$

which lead to

$$
x^{n} \leqslant\left(N \mathrm{e}^{\frac{x-N}{N}}\right)^{n}
$$

$$
\frac{1}{n !} \int_{0}^{\infty} \mathrm{e}^{-x} x^{n} \mathrm{~d} x<\frac{\exp \left[-N\left(1-\frac{n}{N}+\frac{n}{N} \ln \frac{n}{N}\right)\right]}{1-\frac{n}{N}} \leqslant \frac{\exp [-N(1-y+y \ln y)]}{1-y}
$$

(note that $1-(n / N)+(n / N) \ln (n / N)$ is positive and a decreasing function of $n / N$ in the range of interest $0<n<N)$. When $N$ is large enough, the right-hand side of (2.10) is smaller than unity and

$$
\left|\frac{1}{N} \sum_{n=1}^{N y} \ln \left(1-\frac{1}{n !} \int_{N}^{\infty} \mathrm{e}^{-x} x^{n} \mathrm{~d} x\right)\right|<y\left|\ln \left\{1-\frac{\exp [-N(1-y+y \ln y)]}{1-y}\right\}\right| .
$$

When $N$ goes to infinity, the limit of the right-hand side of (2.11) is zero, and therefore the first class gives no contribution to (2.7).

For computing the contribution of the second class, we use

$$
\frac{1}{n !} \int_{N}^{\infty} \mathrm{e}^{-x} x^{n} \mathrm{~d} x<\frac{1}{n !} \int_{n}^{\infty} \mathrm{e}^{-x} x^{n} \mathrm{~d} x=\frac{1}{2}+O\left(n^{-1 / 2}\right)
$$

(the equality in (2.12) is an asymptotic formula in the theory of the incomplete gamma function [7]). Thus, the contribution of the second class is absolutely bounded by

$$
\left|\frac{1}{N} \sum_{n=N y+1}^{N-1} \ln \left(1-\frac{1}{n !} \int_{n}^{\infty} \mathrm{e}^{-x} x^{n} \mathrm{~d} x\right)\right| \sim(1-y) \ln 2 ;
$$

this quantity can be made arbitrarily small by choosing $1-y$ small enough. Therefore, (2.7) does vanish.

Discarding $Q^{\prime}$ in (2.6), we obtain

$$
\frac{1}{N} \ln Q_{\mathrm{exc}} \sim \frac{3}{4} N-\ln \frac{R}{L}-\frac{N+1}{2} \ln N+\frac{1}{N} \sum_{n=1}^{N} \ln n ! .
$$

Since

the sum in (2.14) is

$$
\ln n !=\ln [n \Gamma(n)]=\left(n+\frac{1}{2}\right) \ln n-n+\frac{1}{2} \ln 2 \pi+O\left(\frac{1}{n}\right)
$$

$$
\sum_{n=1}^{N} \ln n !=\sum_{n=1}^{N} \ln n^{n}+\frac{1}{2} \ln N !-\frac{N(N+1)}{2}+\frac{N}{2} \ln 2 \pi+O(\ln N),
$$

where

$$
\sum_{n=1}^{N} \ln n^{n}=\ln \frac{(N !)^{N+1}}{1 ! 2 ! \ldots N !}=(N+1) \ln N !-\sum_{n=1}^{N} \ln n ! .
$$

Combining (2.16) and (2.17), we find

$$
\sum_{n=1}^{N} \ln n !=\frac{2 N+3}{4} \ln N !-\frac{N(N+1)}{4}+\frac{N}{4} \ln 2 \pi+O(\ln N) .
$$


Using the expression (2.15) for $\ln N$ ! in (2.18), and carrying (2.18) into (2.14), we obtain

$$
\frac{1}{N} \ln Q_{\mathrm{exc}} \sim \ln \frac{N^{1 / 2} L}{R}-1+\frac{1}{2} \ln 2 \pi+O\left(\frac{\ln N}{N}\right) .
$$

The temperature is at $k_{\mathrm{B}} T=e^{2} / 2$. Thus, we obtain for the excess free energy per particle in the thermodynamic limit

$$
\begin{aligned}
\frac{F_{\mathrm{exc}}}{N}=-\frac{e^{2}}{2 N} \ln Q_{\mathrm{exc}} & =-\frac{e^{2}}{4} \ln \pi \rho L^{2}+e^{2}\left(\frac{1}{2}-\frac{1}{4} \cdot \ln 2 \pi\right) \\
& =-\frac{e^{2}}{4} \ln \pi \rho L^{2}+0.0405 e^{2} .
\end{aligned}
$$

We now turn to studying the low-temperature properties.

3. Harmonic solid. - 3.1. LATTICE ENERGY. - At zero temperature, the particles organize themselves in a triangular lattice, the energy of which has previously been computed by other authors [3]; they compared an approximation of independent WignerSeitz cells with computer results. Here, we calculate the lattice energy, without a computer, by a simple extension of the Ewald summation method to the case of a logarithmic potential. There are two possible approaches.

One approach is to use for the logarithmic potential the integral representation

$$
-\ln \frac{r}{L}=\frac{1}{2} \int_{0}^{\infty} \frac{\mathrm{e}^{-t r^{2}}-\mathrm{e}^{-t L^{2}}}{t} \mathrm{~d} t,
$$

and proceed afterwards in the usual way with the aid of the transformation

$$
\sum_{\mathbf{R}} \mathrm{e}^{-t R^{2}}=\frac{\pi \rho}{t} \sum_{\mathbf{G}} \mathrm{e}^{-G^{2} / 4 t}
$$

where the sum on $\mathbf{R}$ is on the direct lattice and the sum on $\mathbf{G}$ on the reciprocal lattice. The calculation is very similar to the one which has been done in the case of the $1 / r$ potential in two dimensions [8], and we shall not go into the detail. One must however be careful about a delicate point. In the case of the three-dimensional Coulomb potential, an error has been recently discovered [9] in the usual way of applying the Ewald method, but a second error was also discovered $[10,11]$, and they happen to cancell one another; therefore the usual « careless » treatment gives the correct answer for the lattice energy. We have checked that it is exactly the same situation which occurs in the present case of a two-dimensional Coulomb potential.

Another approach is to consider the logarithmic potential as the limit of a power law by writing

$$
-\ln \frac{r}{L}=\lim _{\varepsilon \rightarrow 0} \frac{1}{\varepsilon}\left[\left(\frac{L}{r}\right)^{\varepsilon}-1\right],
$$

and to use the Ewald method for the potential $1 / r^{\varepsilon}$; the limit $\varepsilon \rightarrow 0$ is taken at the end of the calculation. In three dimensions, there are no difficulties with the Ewald method for an $1 / r^{1+\varepsilon}$ potential [12], and we have checked that this is also the case for an $1 / r^{\varepsilon}$ potential in two dimensions.

Both approaches give for the energy per particle in a perfect lattice

$$
\frac{E_{\mathrm{L}}}{N}=\frac{e^{2}}{4}\left[-\ln \pi \rho L^{2}-1-C-\sum_{\mathbf{R}(\neq 0)} \operatorname{Ei}\left(-\pi \rho R^{2}\right)+\sum_{\mathbf{R}(\neq 0)} \frac{\exp \left(-\pi \rho R^{2}\right)}{\pi \rho R^{2}}\right]
$$

where $C=0.5772 \ldots$ is Euler's constant and $E i$ is the exponential integral function; the sums on $\mathbf{R}$ run on the direct lattice. The second sum in (3.4) comes from a sum on the reciprocal lattice which was made isomorphic to a sum on the direct lattice by a proper choice of the Ewald separation parameter [8]. The fastly convergent sums are easily computed (the number density $\rho$ is related to the lattice spacing $a_{0}$ by $\left.\rho^{-1}=(\sqrt{3} / 2) a_{0}^{2}\right)$, with the result

$$
\frac{E_{\mathrm{L}}}{N}=-\frac{e^{2}}{4} \ln \pi \rho L^{2}-0.37438 e^{2}
$$

This exact result is very well reproduced by the independent hexagonal cell approximation [3] which gives $-0.373412 e^{2}$ instead of the last term of (3.5), or even the simple circular cell approximation which gives $-0.375 e^{2}$; the result from the computer [3], $-0.36433 e^{2}$, is somewhat less accurate ! 
3.2 Phonon SPECTRUM. - We now study the thermal vibrations of the lattice, which should be well described at low temperature by the simple harmonic model : the potential energy is expanded to second order in powers of the displacements from the lattice sites.

The $2 \times 2$ dynamical matrix is easily found to be

$$
D_{\mu v}(\mathbf{k})=\frac{\pi e^{2} \rho}{m} \delta_{\mu v}+\frac{e^{2}}{m} \sum_{\mathbf{R}(\neq 0)}(1-\cos \mathbf{k} . \mathbf{R}) \frac{2 R_{\mu} R_{v}-R^{2} \delta_{\mu v}}{R^{4}},
$$

where $\mu, v=1,2$ label the cartesian axes; $\mathbf{k}$ is the phonon wavenumber. The eigenvalues of this matrix are the squared phonon frequencies. The first term in (3.6) is the contribution from the background.

The sixfold symmetry of the lattice allows simplifications in the lattice sums such as the one occurring in (3.6). Consider for instance a term which depends on the direction of $\mathbf{R}$ only by a factor $R_{x} R_{x}$ (where $R_{x}$ is the component of $\mathbf{R}$ along some $x$-axis). Let $\theta$ be the polar angle of one vector $\mathbf{R}$. In the sum upon $\mathbf{R}$, we can group together the six vectors of same length with polar angles $\theta+n \pi / 3(0 \leqslant n \leqslant 5)$, and we obtain a contribution

$$
\sum_{n=0}^{5} R^{2} \cos ^{2}\left(\theta+n \frac{\pi}{3}\right)=3 R^{2}
$$

as if $R_{x} R_{x}$ had been replaced by $\frac{1}{2} R^{2}$ before the summation. More generally, $R_{\mu} R_{v}$ can be replaced by $\frac{1}{2} R^{2} \delta_{\mu \nu}$. Therefore, the 1 in (3.6) can be omitted.

It is apparent at once that the two phonon frequencies $\omega_{1}(\mathbf{k})$ and $\omega_{2}(\mathbf{k})$ obtained from (3.6) obey the twodimensional version of Kohn's sum rule

$$
\omega_{1}^{2}+\omega_{2}^{2}=\operatorname{Tr} D=\omega_{\mathrm{p}}^{2},
$$

where

$$
\omega_{\mathrm{p}}=\left(2 \pi \rho e^{2} / m\right)^{1 / 2}
$$

is the two-dimensional plasma frequency.

For computing the phonon frequencies themselves, we transform the sum in (3.6) by the Ewald method, in the same way as for the computation of dipolar sums in three dimensions [13]. We obtain

$$
\begin{aligned}
D_{\mu v}(\mathbf{k})=\frac{e^{2}}{m}\left\{(\pi \rho-\alpha) \delta_{\mu \nu}+2 \pi \rho \sum_{\mathbf{G}} \frac{(G+k)_{\mu}(G+k)_{v}}{(\mathbf{G}+\mathbf{k})^{2}} \mathrm{e}^{-(\mathbf{G}+\dot{\mathbf{k}})^{2} / 4 \alpha}+\right. & \\
& \left.+\sum_{\mathbf{R}(\neq 0)}\left[\frac{R^{2} \delta_{\mu \nu}-2 R_{\mu} R_{v}}{R^{4}}-2 \alpha \frac{R_{\mu} R_{v}}{R^{2}}\right] \mathrm{e}^{-\alpha R^{2}} \cos \mathbf{k} \cdot \mathbf{R}\right\},
\end{aligned}
$$

where $\alpha$ is an arbitrary Ewald separation parameter; the sum on $\mathbf{G}$ runs on the reciprocal lattice, the sum on $\mathbf{R}$ runs on the direct lattice.

For studying the behaviour of $D_{\mu v}(\mathbf{k})$ for small values of $k$, it is convenient to go to the limit $\alpha \rightarrow \infty$. With this choice of the Ewald parameter, the sum on $\mathbf{R}$ in (3.10) vanishes. In the sum on $\mathbf{G}$, the term $\mathbf{G}=0$ gives a singular contribution which must be singled out; the other terms can be expanded with respect to $\mathbf{k}$. We have already seen that the sixfold symmetry of the lattice allows the replacement of $G_{\mu} G_{v}$ by $\frac{1}{2} G^{2} \delta_{\mu \nu}$; a similar argument shows that a term $G_{\mu} G_{v}(\mathbf{G . k})^{2}$, which occurs in the expansion with respect to $\mathbf{k}$, can be replaced by $\frac{1}{4} G^{4} k_{\mu} k_{v}+\frac{1}{8} G^{4} k^{2} \delta_{\mu v}$. To order $k^{2}$, we find

$$
\begin{aligned}
D_{\mu v}(\mathbf{k}) \sim \frac{e^{2}}{m} \lim _{\alpha \rightarrow \infty}\left\{(\pi \rho-\alpha) \delta_{\mu \nu}+\right. & 2 \pi \rho \frac{k_{\mu} k_{v}}{k^{2}} \mathrm{e}^{-k^{2} / 4 \alpha}+ \\
& \left.+2 \pi \rho \sum_{\mathbf{G}(\neq 0)}\left[\frac{1}{2} \delta_{\mu v}-\frac{k_{\mu} k_{v}}{4 \alpha}+\frac{G^{2}}{64 \alpha^{2}}\left(2 k_{\mu} k_{v}+k^{2} \delta_{\mu v}\right)\right] \mathrm{e}^{-G^{2} / 4 \alpha}\right\} .
\end{aligned}
$$

In the sum in (3.11), the term $\mathbf{G}=0$ can be added and subtracted, and the unrestricted sum on $\mathbf{G}$ can be replaced by an integral. Since the area of the first Brillouin zone is $4 \pi^{2} \rho$,

$$
\sum_{\mathbf{G}} \rightarrow \frac{1}{4 \pi^{2} \rho} \int \mathrm{d}^{2} G
$$


This replacement introduces an error which vanishes exponentially in the limit $\alpha \rightarrow \infty$. We obtain the small- $k$ behaviour

$$
D_{\mu v}(\mathbf{k}) \sim \omega_{\mathrm{p}}^{2} \frac{k_{\mu} k_{v}}{k^{2}}+\frac{e^{2}}{8 m}\left(k^{2} \delta_{\mu \nu}-2 k_{\mu} k_{v}\right)
$$

Note the singularity at $\mathbf{k}=0$. The dynamical matrix (3.13) is diagonal if $\mathbf{k}$ is chosen as one of the cartesian axes. Its eigenmodes are a plasmon-like longitudinal phonon, with a frequency $\omega_{1}$ given by

$$
\omega_{1}^{2}=\omega_{\mathrm{p}}^{2}-c^{2} k^{2},
$$

and a sound-like transverse phonon, with a frequency

$$
\omega_{\mathrm{t}}=c k,
$$

where

$$
c=\left(\frac{e^{2}}{8 m}\right)^{1 / 2} \text {. }
$$

This behaviour resembles the three-dimensional one. However, the present two-dimensional case has a special feature : the transverse sound velocity $c$ has a simple expression, given by (3.16).

Using (3.10), we can compute the phonon frequencies at different points in the first Brillouin zone depicted in figure 1 . At the point $\mathbf{J}$, it is again convenient to choose the Ewald parameter $\alpha$ as infinite, and one finds $\omega_{1}^{2}=\omega_{\mathrm{t}}^{2}=\omega_{\mathrm{p}}^{2} / 2$; this simple result is a consequence of the threefold symmetry of the reciprocal lattice with respect to $\mathrm{J}$, which lies at the centre of a primitive cell. For other points in the Brillouin zone, a convenient choice of the Ewald parameter is $\alpha=\pi \rho$. At the point X, one finds $\omega_{1}^{2}=0.906 \omega_{\mathrm{p}}^{2}, \omega_{\mathrm{t}}^{2}=0.094 \omega_{\mathrm{p}}^{2}$. The variation of the phonon frequencies along the lines $\Gamma \mathrm{J}, \Gamma \mathrm{X}, \mathrm{JX}$, is sketched in figure 2 . The phonon spectrum does not depend much on the direction of $\mathbf{k}$.
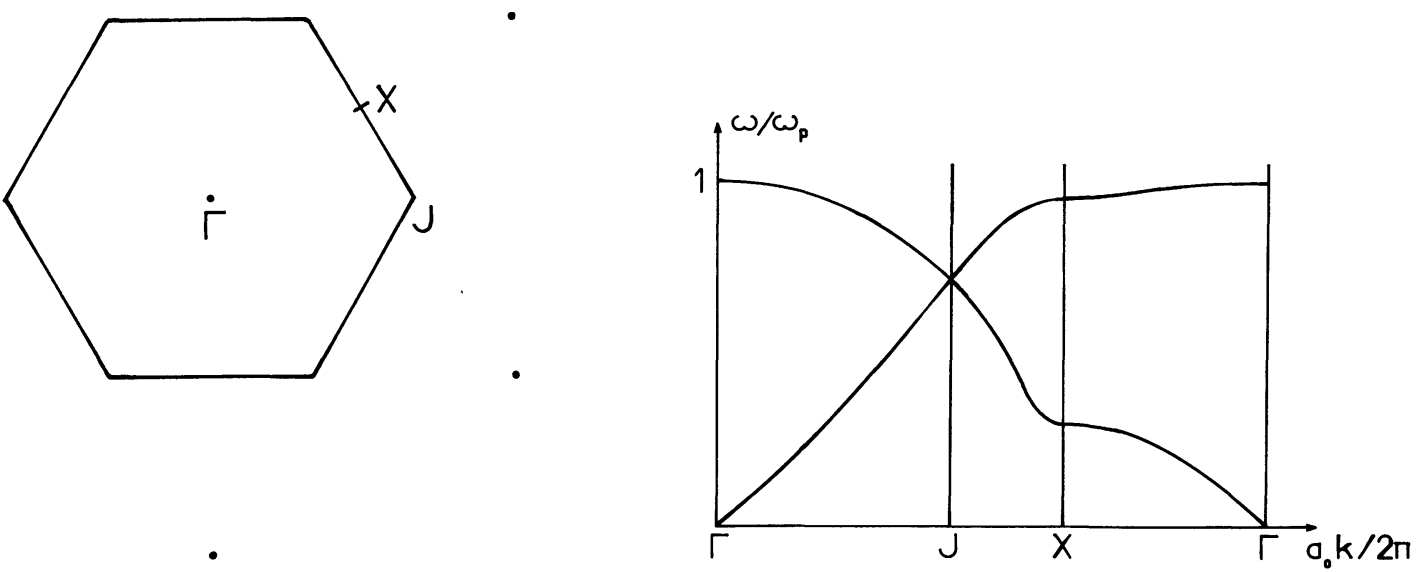

Fig. 1. - The first Brillouin zone. The dots are nodes of the reciprocal lattice. $\Gamma \mathrm{J}=\frac{2}{3} \frac{2 \pi}{a_{0}}, \Gamma \mathrm{X}=\frac{\sqrt{3}}{3} \frac{2 \pi}{a_{0}}\left(a_{0}\right.$ is the direct-lattice

Fig. 2. - Phonon dispersion curves along $\Gamma \mathrm{J}, \Gamma \mathrm{X}, \mathrm{JX}$. spacing).

The essential result here is that the phonon frequencies are everywhere real. Therefore, the triangular lattice is dynamically stable in the domain of validity of the harmonic approximation, i.e. at low temperatures. This is a strong indication that the model is in a crystalline phase at low temperatures. Since, however, the phonon spectrum has an acoustic branch (3.15), the mean square displacement of a particle diverges, and the " crystalline " phase actually is a pseudosolid, with Bragg peaks in the structure factor but no positional long-range order [14]. 
3.3 FREE ENERGY. - In the harmonic approximation, the excess free energy $F_{\text {exc }}^{0}$ is obtained by adding the lattice energy $E_{\mathrm{L}}$ to the vibrational free energy and subtracting the free energy of a perfect gas. One finds

$$
F_{\mathrm{exc}}^{0}=E_{\mathrm{L}}+N k_{\mathrm{B}} T+\frac{1}{2} k_{\mathrm{B}} T \sum_{\mathbf{k s}} \ln \frac{m \omega_{\mathrm{s}}^{2}(\mathbf{k})}{2 \pi \rho k_{\mathrm{B}} T},
$$

where $\omega_{s}(\mathbf{k})$ is the frequency of a phonon of wavenumber $\mathbf{k}$ and polarization $\mathbf{s}$; the sum on $\mathbf{k}$ is on the first Brillouin zone. We approximate the phonon spectrum by the isotropic forms (3.14) and (3.15), and the first Brillouin zone by a disc of same area $4 \pi^{2} \rho$. We then obtain

$$
\begin{aligned}
\frac{F_{\mathrm{exc}}^{0}}{N} & =\frac{E_{\mathrm{L}}}{N}+k_{\mathrm{B}} T\left(2 \ln 2-\frac{3}{2} \ln 3+\ln \frac{e^{2}}{k_{\mathrm{B}} T}\right) \\
& =-\frac{e^{2}}{4} \ln \pi \rho L^{2}+e^{2}\left(-0.37438+\frac{k_{\mathrm{B}} T}{e^{2}} \ln \frac{e^{2}}{k_{\mathrm{B}} T}-0.262 \frac{k_{\mathrm{B}} T}{e^{2}}\right) .
\end{aligned}
$$

We now turn to an improvement of the harmonic approximation, by treating the residual interaction by first-order perturbation theory. Calling $H$ the exact hamiltonian which corresponds to the exact Coulomb interaction (1.1) and $H_{0}$ the hamiltonian of the harmonic approximation, we approximate the excess free energy by

$$
F_{\text {exc }}=F_{\text {exc }}^{0}+\left\langle H-H_{0}\right\rangle_{0},
$$

where $F_{\mathrm{exc}}^{0}$ is the harmonic approximation (3.18) and the average $\left\langle H-H_{0}\right\rangle_{0}$ is taken with the distribution function $\exp \left(-H_{0} / k_{\mathrm{B}} T\right)$. For dealing with the difference $H-H_{0}$, it is enough to consider the displacements of the particles from the lattice sites. Thus

$$
\left\langle H-H_{0}\right\rangle_{0}=\sum_{i>j}\left[\left\langle v\left(\mathbf{R}_{i j}+\mathbf{u}_{j}-\mathbf{u}_{i}\right)\right\rangle_{0}-v\left(R_{i j}\right)\right]+\frac{\pi \rho e^{2}}{2} \sum_{i}\left\langle u_{i}^{2}\right\rangle_{0}-N k_{\mathrm{B}} T,
$$

where $\mathbf{u}_{i}=\mathbf{r}_{i}-\mathbf{R}_{i}$ is the displacement of the $i$-th particle ; the first term in (3.20) comes from the particle-particle interactions, the second term comes from the particle-background interactions, and the third term comes from the subtraction of the harmonic potential energy.

The fluctuations of $\mathbf{u}_{i j}=\mathbf{u}_{j}-\mathbf{u}_{i}$ are gaussian. We label $\| \mathbf{a}$ component along $\mathbf{R}_{i j}$ and $\perp$ a component normal to $\mathbf{R}_{i j}$. It results from the symmetry of the lattice that $u_{i j \|}$ and $u_{i j \perp}$ are independent variables. Let $\lambda_{\|}$ and $\lambda_{\perp}$ be their mean square values :

$$
\lambda_{\|}\left(R_{i j}\right)=\left\langle\left(u_{i j \|}\right)^{2}\right\rangle_{0}, \quad \lambda_{\perp}\left(R_{i j}\right)=\left\langle\left(u_{i j \perp}\right)^{2}\right\rangle_{0} .
$$

In the harmonic approximation, the average potential energy of vibration obeys

$$
\sum_{i>j}\left[\frac{1}{2} \nabla_{\|}^{2} v\left(R_{i j}\right) \lambda_{\|}\left(R_{i j}\right)+\frac{1}{2} \nabla_{\perp}^{2} v\left(R_{i j}\right) \lambda_{\perp}\left(R_{i j}\right)\right]+\frac{\pi \rho e^{2}}{2} \sum_{i}\left\langle u_{i}^{2}\right\rangle_{0}=N k_{\mathrm{B}} T,
$$

where $\nabla_{\|}$and $\nabla_{\perp}$ are the components of the gradient along $\mathbf{R}_{i j}$ and normal to it, respectively. The explicit form of the second derivatives is, from (1.1),

$$
\nabla_{\|}^{2} v(R)=\frac{e^{2}}{R^{2}}, \quad \nabla_{\perp}^{2} v(R)=-\frac{e^{2}}{R^{2}} .
$$

Using (3.22) and (3.23), we can recast (3.20) into a more convenient form

where

$$
\left\langle H-H_{0}\right\rangle_{0}=\sum_{i>j}\left\{\psi\left(R_{i j}\right)+\frac{1}{2}\left[\lambda_{\perp}\left(R_{i j}\right)-\lambda_{\|}\left(R_{i j}\right)\right] \frac{e^{2}}{R_{i j}^{2}}\right\},
$$

$$
\psi\left(\boldsymbol{R}_{i j}\right)=\left\langle v\left(\mathbf{R}_{i j}+\mathbf{u}_{i j}\right)\right\rangle_{0}-v\left(\boldsymbol{R}_{i j}\right) .
$$

In two dimensions, the mean square displacement of a particle $\left\langle u_{i}^{2}\right\rangle_{0}$, which occurs in (3.20), diverges; this divergence is cancelled by another divergence in the first term of (3.20), but manipulating these divergences would be unconvenient. The form (3.24) has the advantage of being free from divergences, since the sum on $\mathbf{R}$ in (3.24) converges (see eq. (3.35)). 
We need the average displacements (3.21). Expressing these displacements in terms of phonon variables, the mean squares of which are obtained by using the equipartition of energy, we find

$$
\lambda_{\|}(R)=\frac{2}{N} \sum_{\mathbf{k}, \mathbf{s}}(1-\cos \mathbf{k} \cdot \mathbf{R}) \frac{k_{\mathrm{B}} T}{m \omega_{\mathrm{s}}^{2}(\mathbf{k})}\left[\hat{\mathbf{e}}_{\mathrm{s}}(\mathbf{k}) \cdot \hat{\mathbf{R}}\right]^{2},
$$

where $\hat{\mathbf{e}_{\mathbf{s}}}(\mathbf{k})$ is the unit polarization vector of the phonon $(\mathbf{k}, \mathbf{s})$ and $\hat{\mathbf{R}}$ the unit vector along $\mathbf{R}$; similarly,

$$
\lambda_{\perp}(R)=\frac{2}{N} \sum_{\mathbf{k}, \mathbf{s}}(1-\cos \mathbf{k} \cdot \mathbf{R}) \frac{k_{\mathrm{B}} T}{m \omega_{\mathrm{s}}^{2}(\mathbf{k})}\left[\hat{\mathbf{e}}_{\mathrm{s}}(\mathbf{k}) \wedge \hat{\mathbf{R}}\right]^{2} .
$$

Since most of the fluctuations come from the small- $k$ transverse phonons, we approximate here the phonon spectrum simply by transverse phonons with a frequency $\omega_{\mathrm{t}}=c k$ and longitudinal phonons with a frequency $\omega_{1}=\omega_{\mathrm{p}}$, in a circular Brillouin zone of area $4 \pi^{2} \rho$. We obtain

$$
\lambda_{\|}=\frac{k_{\mathrm{B}} T}{\pi \rho e^{2}}\left[4 \int_{0}^{x} \frac{1-J_{0}(x)}{x} \mathrm{~d} x-\frac{3}{2}+2 \frac{J_{1}(x)}{x}+2 \frac{1-J_{0}(x)}{x^{2}}\right],
$$

where $x=2(\pi \rho)^{1 / 2} R_{i j}$ (the $J$ are Bessel functions), and

$$
\lambda_{\perp}=\lambda_{\|}+\frac{k_{\mathrm{B}} T}{\pi \rho e^{2}}\left[4-6 \frac{J_{1}(x)}{x}-4 \frac{1-J_{0}(x)}{x^{2}}\right] .
$$

The integral in (3.28) can be evaluated by using the series expansion of $J_{0}(x)$ and the asymptotic form

$$
\int_{0}^{x} \frac{1-J_{0}(x)}{x} \mathrm{~d} x \underset{x \rightarrow \infty}{\sim} \ln x+C-\ln 2 .
$$

We now compute the average potential which occurs in $(3.25)$ :

$$
\psi(R)=-e^{2}\left\langle\ln \frac{\left[\left(R+u_{\|}\right)^{2}+u_{\perp}^{2}\right]^{1 / 2}}{L}\right\rangle_{0}+e^{2} \ln \frac{R}{L} .
$$

Changing from the gaussian variables $u_{\|}$and $u_{\perp}$ to polar variables $v, \theta$ defined by

$$
\frac{u_{\|}}{R}=v \cos \theta, \quad \frac{u_{\perp}}{R}=\left(\frac{\lambda_{\perp}}{\lambda_{\|}}\right)^{1 / 2} v \sin \theta,
$$

we obtain

$$
\psi(R)=-\frac{e^{2} R^{2}}{2 \lambda_{\|}} \int_{0}^{\infty} \mathrm{d} v v \mathrm{e}^{-R^{2} v^{2} / 2 \lambda_{\|}} \frac{1}{2 \pi} \int_{0}^{2 \pi} \mathrm{d} \theta \ln \left[(1+v \cos \theta)^{2}+\frac{\lambda_{\perp}}{\lambda_{\|}} v^{2} \sin ^{2} \theta\right]
$$

It should be noted that $\lambda_{\perp} / \lambda_{\|}>1$. The integral on $\theta$ is performed by writing the argument of the logarithm as a product of terms of the form $A+B \cos \theta$. After some manipulations, we find

$$
\psi(R)=\frac{e^{2}}{2}\left\{\operatorname{Ei}\left(-\frac{R^{2}}{2 \lambda_{\|}}\right)-\int_{0}^{1} \frac{\mathrm{d} z}{z} \mathrm{e}^{-R^{2} z / 2 \lambda_{\|}}\left[1-\left(1+\frac{\lambda_{\perp}-\lambda_{\|}}{\lambda_{\|}} z\right)^{-1 / 2}\right]\right\} .
$$

The integral in (3.34) must be performed numerically. This integral would not occur if the anisotropy factor $\left(\lambda_{\perp}-\lambda_{\|}\right) / \lambda_{\|}$could be neglected, but we have checked that this approximation would be too drastic. Keeping this integral, we find that, for large $R$ values, the summand in (3.24) decreases as

$$
\psi(R)+\frac{e^{2}\left(\lambda_{\perp}-\lambda_{\|}\right)}{2 R^{2}} \underset{R \rightarrow \infty}{\sim} \frac{12\left(k_{\mathrm{B}} T\right)^{2}}{e^{2}\left(\pi \rho R^{2}\right)^{2}} .
$$

Using (3.28), (3.29), (3.34), we can compute the correction (3.24) to the free energy. This correction of course becomes important only at high enough temperatures. We have computed it explicitly only at $k_{\mathrm{B}} T=e^{2} / 2$. The result will be discussed in section 5 . 
4. Self-consistent harmonic approximation. - As the temperature increases, the simple harmonic approximation becomes less and less reliable, even if we include the correction (3.19). A better approximation is the self-consistent harmonic approximation (SCHA); it has been used for three-dimensional [15] and two-dimensional [16] electron solids. An especially attractive feature of the SCHA is that it may provide information about the stability of the solid phase, as we shall see.

The SCHA is a variational approach. One uses a trial harmonic hamiltonian $H_{0}$ describing a collection of phonons, the frequencies and polarizations of which are now treated as free parameters. One expresses the free energy by the approximate formula (3.19), which is an upper bound for the free energy of the crystalline model (see however the Appendix). The parameters of $H_{0}$ are then determined by minimizing (3.19).

Let us minimize (3.19) with respect to one phonon frequency $\omega_{s}(\mathbf{k})$. Noting that

$$
\left\langle v\left(\mathbf{R}_{i j}+\mathbf{u}_{i j}\right)\right\rangle_{0}=\left\langle\exp \left(\mathbf{u}_{i j} \cdot \nabla\right)\right\rangle_{0} v\left(R_{i j}\right)=\exp \left(\frac{1}{2} \lambda_{\|} \nabla_{\|}^{2}+\frac{1}{2} \lambda_{\perp} \nabla_{\perp}^{2}\right) v\left(R_{i j}\right),
$$

and that

$$
\sum_{i}\left\langle u_{i}^{2}\right\rangle_{0}=\frac{k_{\mathrm{B}} T}{m} \sum_{\mathbf{k s}} \frac{1}{\omega_{\mathrm{s}}^{2}(\mathbf{k})}
$$

we can rewrite formally $(3.20)$ as

$$
\left\langle H-H_{0}\right\rangle_{0}=\sum_{i>j}\left[\exp \left(\frac{1}{2} \lambda_{\|} \nabla_{\|}^{2}+\frac{1}{2} \lambda_{\perp} \nabla_{\perp}^{2}-1\right)\right] v\left(R_{i j}\right)+\frac{k_{\mathrm{B}} T \omega_{\mathbf{p}}^{2}}{4} \sum_{\mathbf{k s}} \frac{1}{\omega_{\mathbf{s}}^{2}(\mathbf{k})}-N k_{\mathrm{B}} T
$$

where the first term depends on $\omega_{s}(\mathbf{k})$ through the expressions (3.26) and (3.27) of $\lambda_{\|}$and $\lambda_{\perp}$. Using (3.17) and (4.3) in (3.19), from

$$
\frac{\partial F_{\mathrm{exc}}}{\partial \omega_{\mathrm{s}}(\mathbf{k})}=0
$$

we obtain

$$
\omega_{\mathrm{s}}^{2}(\mathbf{k})=\frac{1}{2} \omega_{\mathrm{p}}^{2}+\frac{1}{m} \sum_{\mathbf{R}(\neq 0)}(1-\cos \mathbf{k} \cdot \mathbf{R})\left[\left(\hat{\mathbf{e}}_{\mathrm{s}} \cdot \hat{\mathbf{R}}\right)^{2} \nabla_{\|}^{2}+\left(\hat{\mathbf{e}}_{\mathrm{s}} \wedge \hat{\mathbf{R}}\right)^{2} \nabla_{\perp}^{2}\right][\psi(R)+v(R)]
$$

This equation (4.5) determines self-consistently the best spectrum $\omega_{\mathbf{s}}(\mathbf{k})$.

Here, we use a simplified version of the SCHA, with only one free parameter, namely the transverse-sound velocity. We look for a transverse mode with a small- $k$ behaviour

$$
\omega_{\mathbf{s}}^{2}(\mathbf{k})=\xi c^{2} k^{2}, \quad \hat{\mathbf{e}}_{\mathbf{s}} \cdot \mathbf{k}=0,
$$

where $c$ is the simple harmonic sound velocity $(3.16)$ and $\xi$ the variational parameter. In the simple harmonic approximation, the transverse matrix element of the dynamical matrix (3.6) is, at small- $k$-values,

$$
c^{2} k^{2}=\frac{1}{2} \omega_{\mathrm{p}}^{2}+\frac{1}{m} \sum_{\mathbf{R}(\neq 0)}(1-\cos \mathbf{k} . \mathbf{R})\left[\left(\hat{\mathbf{e}}_{\mathrm{s}} \cdot \hat{\mathbf{R}}\right)^{2} \nabla_{\|}^{2}+\left(\hat{\mathbf{e}}_{\mathrm{s}} \wedge \hat{\mathbf{R}}\right)^{2} \nabla_{\perp}^{2}\right] v(R) .
$$

Subtracting (4.7) from (4.5), and expanding cos k.R to order $k^{2}$, we obtain

$$
\xi-1=\frac{1}{2 m c^{2}} \sum_{\mathbf{R}(\neq 0)} R^{2} \cos ^{2} \theta\left(\sin ^{2} \theta \nabla_{\|}^{2}+\cos ^{2} \theta \nabla_{\perp}^{2}\right) \psi(\mathrm{R}),
$$

where $\theta$ is the angle between $\mathbf{k}$ and $\mathbf{R}$. In the lattice sum in (4.8), the sixfold symmetry allows the replacement of $\cos ^{2} \theta \sin ^{2} \theta$ by $1 / 8$ and $\cos ^{4} \theta$ by $3 / 8$. Using

$$
\nabla_{\|}^{2} \psi=\frac{\partial^{2} \psi}{\partial R^{2}}, \quad \nabla_{\perp}^{2} \psi=\frac{1}{R} \frac{\partial \psi}{\partial R},
$$

we finally obtain the equation which determines the parameter $\xi$ :

$$
\xi-1=F(\xi)
$$


where

$$
\begin{aligned}
F(\xi) & =\frac{1}{2 e^{2}} \sum_{\mathbf{R}(\neq 0)} \frac{1}{R} \frac{\partial}{\partial R}\left(R^{3} \frac{\partial \psi}{\partial R}\right) \\
& =\sum_{\mathbf{R}(\neq 0)}\left[1+\frac{R^{2}}{2 \lambda_{\|}} \int_{0}^{1} \mathrm{~d} z \mathrm{e}^{-R^{2} z / 2 \lambda_{\|}}\left(\frac{R^{2}}{2 \lambda_{\|}} z-2\right)\left(1+\frac{\lambda_{\perp}-\lambda_{\|}}{\lambda_{\|}} z\right)^{-1 / 2}\right] .
\end{aligned}
$$

The function $F(\xi)$ does depend on $\xi$ through $\lambda_{\|}$and $\lambda_{\perp}$ which are still given by (3.26) and (3.27), where the transverse phonon frequency to be used is now $\xi^{1 / 2} c k$ (we $\operatorname{keep} \omega_{\mathrm{p}}$ for the longitudinal phonon frequency). As a consequence, (3.28) and (3.29) are to be replaced by

$$
\lambda_{\|}=\frac{k_{\mathrm{B}} T}{\pi \rho e^{2}}\left[\frac{4}{\xi} \int_{0}^{x} \frac{1-J_{0}(x)}{x} \mathrm{~d} x+\frac{1}{2}-\frac{2}{\xi}+\left(\frac{4}{\xi}-2\right) \frac{J_{1}(x)}{x}+2 \frac{1-J_{0}(x)}{x^{2}}\right]
$$

and

$$
\lambda_{\perp}=\lambda_{\|}+\frac{k_{\mathrm{B}} T}{\pi \rho e^{2}}\left[\frac{4}{\xi}+\left(2-\frac{8}{\xi}\right) \frac{J_{1}(x)}{x}-4 \frac{1-J_{0}(x)}{x^{2}}\right] .
$$

The solutions of (4.10) can be obtained graphically, by plotting on the same graph the straight line $\xi-1$ and the function $F(\xi)$, as shown in figure 3. The function $F(\xi)$ depends on the temperature. At low temperature, there are two solutions for $\xi$. It can be shown that the larger one corresponds to a minimum of the free energy (the smaller one gives a maximum). At very low temperature, that solution which gives the minimum is close to $\xi=1$, i.e. the simple harmonic model is the best one. As the temperature increases, the curve $F(\xi)$ moves down, and the transverse sound velocity $c \xi^{1 / 2}$ decreases until, above some temperature $T_{0}$, there is no longer a solution to (4.10) : no selfconsistent transverse-sound velocity exists. We consider this as being a strong indication that no solid phase can exist above $T_{0}$. One must however realize that such an approach gives only an upper limit for the melting temperature, since a phase transition may occur before the unstability limit $T_{0}$ is reached. For three- and two-dimensional electron systems, computer simulations $[17,18]$ have indeed located the melting temperature much below the unstability limit deter-

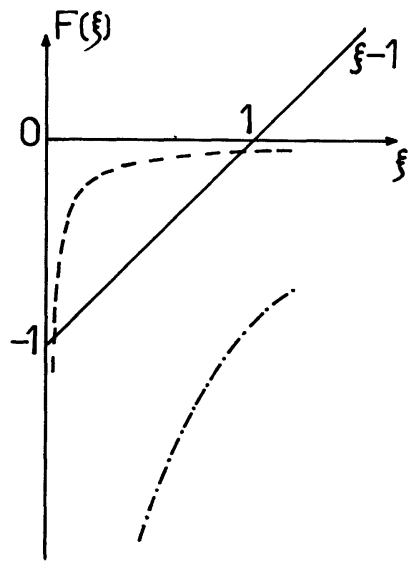

Fig. 3. - Graphical resolution of (4.10). The dashed line is $F(\xi)$ at low temperature. The dashed-dotted line is $F(\xi)$ at $k_{\mathrm{B}} T=e^{2} / 2$. mined by the SCHA $[15,16$. Therefore, we have not attempted to determine $T_{0}$. We have done numerical work only at the special temperature $k_{\mathrm{B}} T=e^{2} / 2$, and have found no solution in that case; thus the system should have already melted at this temperature.

5. Summary and conclusion. - The excess free energy per particle is of the form

$$
\frac{F_{\text {exc }}}{N}=-\frac{e^{2}}{4} \ln \pi \rho L^{2}+e^{2} g(\Gamma),
$$

where $\Gamma=e^{2} / k_{\mathrm{B}} T$. The temperature dependence of the free energy is described by the function $g(\Gamma)$. We have obtained the exact result

$$
g(2)=\frac{1}{2}-\frac{1}{4} \ln 2 \pi=0.0405 .
$$

On the weak-coupling (high-temperature) side, the Debye approximation [4] gives

$$
g(\Gamma)=\frac{1}{4}\left(\ln \frac{2}{\Gamma}+1-2 C\right),
$$

where $C=0.5772 \ldots$ is Euler's constant ; in this approximation, $g(2)=-0.0386$. A more elaborate approximation based on an integral equation [5], which should have a larger domain of validity, gives .

$g(\Gamma)=-\frac{1}{4}\left\{2 C+\ln \left[\frac{\Gamma}{\Gamma+2}\left(\frac{2}{\Gamma+2}\right)^{2 / \Gamma}\right]\right\}$

(there is an error in Eq. (38) of Ref. [5] which contains a spurious 1); this approximation gives $g(2)=0.0580$.

In the present paper, we have studied the strongcoupling (low-temperature) side, using first a harmonic lattice theory. We have found in (3.18)

$$
g(\Gamma)=-0.37438+\frac{\ln \Gamma-0.262}{\Gamma} ;
$$


in this approximation, $g(2)=-0.16$. The improved harmonic approximation (3.19), which treats the residual interaction as a perturbation gives

$$
g(2)=-0.09
$$

(this value is below the exact one (5.2); this point is discussed in the Appendix).

The different results for the free energy are gathered in figure 4. The high-temperature approximation (5.4) everywhere remains above the low-temperature approximation (5.5).

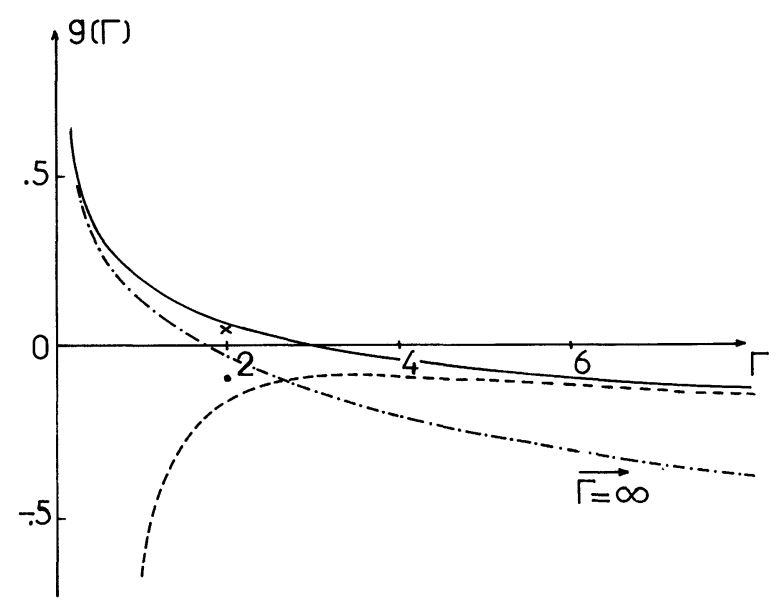

Fig. 4. - The temperature-dependent factor $g(\Gamma)$ of the excess free energy (eq. (5.1)). The dashed-dotted line is the Debye approximation (5.3). The full line is the result (5.4) from the integral equation approach. The dashed line is the harmonic lattice approximation (5.5). At $\Gamma=2$, the cross is the exact result (5.2), the dot is the result from the improved harmonic approximation (3.19). The arrow indicates the strong-coupling limit, which is approached by the harmonic dashed line for large $\Gamma$ values.

The existence of a well-behaved harmonic theory at low temperature, with real phonon frequencies, strongly suggests that the model is in a (pseudo) crystalline phase. On the other hand, the breakdown of the SCHA at some temperature $T_{0}$ (smaller than $e^{2} / 2 k_{\mathrm{B}}$ ) strongly suggests that this crystalline phase does not persist at high temperatures. At $\Gamma=2$, there is a close agreement between the exact $g(2)$ and (5.4); since (5.4) is obtained from an approximate theory built for a fluid phase, this is another indication that there is a fluid phase domain which includes $\Gamma=2$. Therefore, it is very likely that there is at least one phase transition (and perhaps several ones [19]) between the low-temperature solid and a hightemperature fluid, with the unusual feature that the equation of state (1.2) has no singularity anywhere.
Acknowledgments. - We are indebted to C. Deutsch, H. DeWitt and P. Nozières for stimulating discussions.

\section{APPENDIX}

Upper bound to the free energy and particle indistinguishability. - Let $F_{\text {exact }}$ be the exact free energy which corresponds to some hamiltonian $H$. Let $H_{0}$ be some approximate trial hamiltonian, and $F_{0}$ the corresponding free energy. A standard theorem of statistical mechanics states that

$$
F_{0}+\left\langle H-H_{0}\right\rangle_{0} \geqslant F_{\text {exact }},
$$

where $\left\langle H-H_{0}\right\rangle_{0}$ is an average taken with the distribution $\exp \left(-H_{0} / k_{\mathrm{B}} T\right)$.

Here, we used for $H_{0}$ a harmonic lattice model, and found that (A.1) is violated at $\Gamma=2$ (see Fig. 4).

The explanation is that (A.1) is valid only if $F_{\text {exact }}$ and $F_{0}$ are computed with the same normalization of phase space. In classical statistical mechanics, one should compute configuration space integrals as

$$
\frac{1}{N !} \int \mathrm{d} \mathbf{r}_{1} \mathrm{~d} \mathbf{r}_{2} \ldots \mathrm{d} \mathbf{r}_{N} \ldots
$$

where the factor $N$ ! is the classical remnant of the indistinguishability of the particles. This is indeed the way in which $F_{\text {exact }}$ has been computed. In the harmonic approximation, however, the particles are made distinguishable by being attached to different sites, and one uses

$$
\int \mathrm{dr}_{1} \mathrm{~d} \mathbf{r}_{2} \ldots \mathrm{d} \mathbf{r}_{N} \ldots
$$

Therefore (A.1) is not valid. It is not possible, with a harmonic hamiltonian, to put a $1 / N$ ! in (A.3), because the free energy $F_{0}$ would no longer be extensive.

The validity of (A.1) is restored if there is no overlap between the individual distribution functions of the particles. The prescription (A.3) then provides the correct counting, since a configuration in which there is one particle at $\mathbf{r}_{1}$, one particle at $\mathbf{r}_{2}$, etc... is counted only once. At very low temperature, the overlap is indeed negligible, and (A.1) should be satisfied. At higher temperatures, when the individual distribution functions do overlap, the prescription (A.3) overestimates the entropy and may lead to an approximate free energy lower than the exact one. 


\section{References}

[1] Hauge, E. H. and Hemmer, P. C., Phys. Norv. 5 (1971) 209, and references quoted there.

[2] Sari, R. R. and Merlini, D., J. Stat. Phys. 14 (1976) 91.

[3] Sari, R. R., Merlini, D., and Calinon, R., J. Phys. A 9 (1976) 1539.

[4] Deutsch, C., DeWitt, H. E. and Furutani, Y., Phys. Rev. $A 20$ (1979) 2631, and references quoted there.

[5] Calinon, R., Golden, K. I., Kalman, G. and Merlini, D., Phys. Rev. A 20 (1979) 329.

[6] Bakshi, P., Calinon, R., Golden, K. I., Kalman, G., and Merlini, D., Phys. Rev. A 20 (1979) 336.

[7] Erdelyi, A., Higher Transcendental Functions, Vol. 2 (Mc Graw Hill, New York) 1953.

[8] Bonsall, L. and Maradudin, A. A., Phys. Rev. B 15 (1977) 1959.

[9] Hall, G. L., Phys. Rev. B 19 (1979) 3921.
[10] De Wette, F. W., Phys. Rev. B 21 (1980) 3751.

[11] Ihm, J. and Cohen, M. L., Phys. Rev. B 21 (1980) 3754.

[12] Hall, G. L., J. Math. Phys. 21 (1980) 698.

[13] Cohen, M. H. and Keffer, F., Phys. Rev. 99 (1955) 1128 ; erratum, Phys. Rev. B 10 (1974) 787.

[14] Jancovici, B., Phys. Rev. Lett. 19 (1967) 20.

[15] Kugler, A. A., Ann. Phys. 53 (1969) 133. In this reference, the SCHA is called renormalized harmonic approximation (RHA).

[16] Platzman, P. M. and Fukuyama, H., Phys. Rev. B 10 (1974) 3150 .

[17] Pollock, E. L. and Hansen, J. P., Phys. Rev. A 8 (1973) 3110.

[18] Gann, R. C., Chakravarty, S., and Chester, G. V., Phys. Rev. B 20 (1979) 326.

[19] Nelson, D. R. and HalPerin, B. I., Phys. Rev. B 19 (1979) 2457. 